\section{Melanom: Immuntherapie in der Erstlinie?}

\author{
Inwiefern profitieren Patienten mit fortgeschrittenem Melanom von einer \\ Erstlinienbehandlung mit einer Target-Therapie oder einer Immuntherapie? \\ Das wurde anhand einer Netzwerk-Metaanalyse untersucht.
}

Studien, in denen beim fortgeschrittenen Melanom mit BRAF-Mutationen moderne Therapiestrategien direkt verglichen wurden, fehlen. Deshalb haben kanadische Epidemiologen und Onkologen die Daten verfügbarer randomisierter kontrollierter Studien der Phasen II und III mithilfe unter anderem der Netzwerk-Metaanalyse ausgewertet. Diese Methode der statistischen Auswertung von Studiendaten macht Schätzungen von Effekten möglich und gewinnt zunehmend an Bedeutung.

Die jüngsten in der Analyse berücksichtigten Studien waren im April 2016 veröffentlicht worden. Mindestens eine von zwei Therapiestrategien musste Gegenstand der jeweiligen Untersuchung gewesen sein: eine Targeted-Therapie mit BRAF/MEK-Hemmern oder eine Immuntherapie mit Checkpointhemmern gegen PD-1(„programmed death 1 “)- oder CTLA-4(„,ytotoxic T-lymphocyte antigen 4 “)-Rezeptoren.

Die Wissenschaftler richteten ihr Augenmerk auf die Parameter progressionsfreies Überleben (PFS), Gesamtüberleben (OS) und objektive Ansprechrate
(ORR) sowie auf die unerwünschten Wirkungen der jeweiligen Therapie. Für jeden Parameter berechneten sie zudem den Index-Wert SUCRA („surface under the cumulative ranking curve“, 0-100\%). Niedrigste Werte sind ein Hinweis auf die schlechteste Therapie, höchste Werte auf die beste Therapie. Insgesamt 15 randomisierte kontrollierte Studien mit fast 6.700 Patienten waren geeignet.

Im Vergleich zu allen anderen Therapieoptionen - außer Behandlungen mit CTLA-4-Antikörper plus GM-CSF - war der Analyse zufolge das OS mit BRAF/ MEK- oder Checkpointinhibitoren signifikant besser. Die Hazard Ratio (HR) beim Vergleich zwischen BRAF/MEK versus BRAF allein lag bei 0,69 (95\%-KI 0,59-0,82) zuungunsten von BRAF; Patienten unter BRAF/MEK hatten demnach eine um $31 \%$ höhere Überlebensrate. Beim Vergleich PD-1-Hemmung versus CTLA-4-Hemmung errechneten die Wissenschaftler eine $\mathrm{HR}$ von 0,58 (95\%-KI 0,47-0,71). Die Überlebenswahrscheinlichkeit lag hier für die PD1-Inhibition um $42 \%$ höher als in der Vergleichsgruppe. Der Nutzen der bei- den Therapiestrategien spiegelt sich auch im SUCRA-Wert wider: Mit 92\% (BRAF/ MEK-Kombination) und $90 \%$ (PD-1) war mit ihnen die höchste Wahrscheinlichkeit für ein Überleben verknüpft.

Auch bei Betrachtung des Parameters PFS wurden mit den meisten neuen Substanzen die besten SUCRA-Ergebnisse ermittelt, nämlich mit BRAF/MEKHemmern (100\%), PD-1/CTLA-4-Hemmern $(87 \%)$ und BRAF-Hemmern (78\%). Als schlechteste Therapieoption entpuppten sich MEK-Hemmer (7\%).

Mit BRAF/MEK-Hemmern lassen sich schließlich die besten ORR erzielen (SUCRA: $100 \%$ ), gefolgt von BRAFHemmern (87\%) und PD-1/CTLA4-Hemmern (75\%). Darüber hinaus schließen die Wissenschaftler aus der SUCRA-Analyse, dass das Risiko für Nebenwirkungen bei einer Chemotherapie und bei einer Behandlung mit PD1 -Hemmern am geringsten ist (93\%).

Fazit: Patienten mit fortgeschrittenem Melanom profitieren gleichermaßen von einer Erstlinienbehandlung mit einer Target-Therapie oder einer Immuntherapie im Vergleich zu anderen Strategien wie der Chemotherapie. Peter Leiner

Devji T et al. Systemic Therapy for Previously Untreated Advanced BRAF-Mutated

Melanoma - A Systematic Review and Network Meta-Analysis of Randomized Clinical Trials. JAMA Oncol 2017; 3: 366-73

\title{
Ein Glomustumor unter dem Fingernagel
}

Ein 46-jähriger Mann stellte sich in der dermatologischen Klinik vor, weil er seit sieben Jahren immer wieder einmal Schmerzen unter dem Fingernagel des vierten Fingers links empfand. Die Schmerzen traten vor allem bei kaltem Wetter auf. Bei der Untersuchung erkannte man eine druckschmerzhafte, bläuliche Veränderung von circa $3 \mathrm{~mm}$ Durchmesser unter dem Fingernagel. Diese wurde exzidiert.

Histologisch ergab sich ein Glomustumor. Dabei handelt es sich um eine seltene Neoplasie der glatten Muskulatur von Gefäßen, die am häufigsten im Nagelbett von Finger- und Zehennägeln vorkommt. Sie nimmt ihren Ursprung in den Glomus-Körperchen, thermoregulatorischen Strukturen der Dermis. Die exakte Diagnose kann nur histologisch gestellt werden. Allerdings sind subunguale Läsionen, die vor allem bei Kälte schmerzen, pathognomonisch. Es gibt in diesem Fall auch kaum eine Differenzialdiagnose.

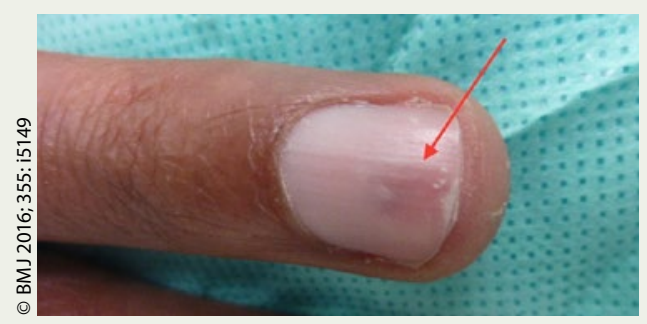

Bläuliche Veränderung unter dem Fingernagel

Schon bei geringer Größe des Tumors können intensive Schmerzen auftreten, die sich durch Analgetika kaum beeinflussen lassen. Die Schmerzen können bereits vorhanden sein, ehe man den Tumor unter dem Nagel überhaupt erkennen kann. Also ein klassischer Fall von daran denken und die richtigen Fragen stellen. Die Therapie besteht in der Exzision der Veränderungen. Prof. Hermann S. FüeßI

George $C$ et al. The case of a glomus tumour. BMJ 2016; 355: i5149 